Arezki Touzaline (Bab-Ezzouar)

\title{
A UNILATERAL CONTACT PROBLEM WITH SLIP-DEPENDENT FRICTION
}

Abstract. We consider a mathematical model which describes a static contact between a nonlinear elastic body and an obstacle. The contact is modelled with Signorini's conditions, associated with a slip-dependent version of Coulomb's nonlocal friction law. We derive a variational formulation and prove its unique weak solvability. We also study the finite element approximation of the problem and obtain an optimal error estimate under extra regularity for the solution. Finally, we establish the convergence of an iterative method to the finite element problem.

1. Introduction. Contact problems involving deformable bodies are quite frequent in industry as well as in daily life and play an important role in structural and mechanical systems. A first attempt to study contact problems within the framework of variational inequalities was made in [11]. The unilateral contact models take an important place in the theory of variational inequalities and the approximation by finite element methods (see [14, 15]). The mathematical analysis of unilateral contact problems, including existence and uniqueness results, was widely developed in [13]. Numerical studies of the Signorini contact problem were made in [2, 4, 3, 15]. The mathematical and the numerical state of the art can be found in [18, 15]. Recently a static contact problem with normal compliance and unilateral constraint associated with a slip-dependent version of Coulomb's law of dry friction was studied in [1] and numerical results were presented.

In this paper we deal with the analysis and numerical approximation of a unilateral contact problem with Signorini's conditions and slip-dependent

2010 Mathematics Subject Classification: 74B20, 74M15, 49J40.

Key words and phrases: nonlinear elasticity, nonlocal friction, variational inequality, approximation.

Received 26 January 2015.

Published online 26 November 2015. 
nonlocal friction law for elastic materials. Historically for the first time Signorini's conditions and the nonlocal friction law first appeared respectively in [17] and [11]. In [6, 16] the static unilateral contact problem with nonlocal friction law was resolved by different numerical approaches, while the quasistatic unilateral contact problem with nonlocal friction law was studied in [9].

We recall that the model of slip-dependent friction is considered in geophysics and solid mechanics corresponding to a smooth dependence of the friction coefficient on the slip $u_{\tau}$, i.e. $\mu=\mu\left(\left|u_{\tau}\right|\right)$. Several authors have been interested in the study of such models: see for instance [8, 10] and the references therein.

The current paper represents a continuation of [19] concerning the study of contact problems with slip-dependent friction. We assume that the elasticity operator is nonlinear, strongly monotone and Lipschitz continuous. We suppose that the displacement field is of class $H^{2}$ (the standard Sobolev space of degree 2). We deduce, under an extra regularity for the solution, an optimal error estimate $O(h)$, where $h>0$ stands for the discretization parameter.

The rest of the paper is structured as follows. In Section 2 the mechanical problem (Problem $P_{1}$ ) is formulated, some notation is presented and the variational formulation is established. In Section 3 we prove an existence and uniqueness result. In Section 4 we study the finite element approximation of the displacement variational formulation. We establish the convergence of the finite element method and derive order error estimates under appropriate regularity assumptions on the solution. We also introduce an iterative method to solve the finite element problem (Problem $P_{h}$ ), which converges under certain assumptions.

2. Problem statement and variational formulation. Consider an elastic body occupying a bounded Lipschitzian domain $\Omega \subset \mathbb{R}^{d}(d=2,3)$. The boundary $\Gamma$ of $\Omega$ is partitioned into three measurable parts such that $\Gamma=\bar{\Gamma}_{1} \cup \bar{\Gamma}_{2} \cup \bar{\Gamma}_{3}$, where $\Gamma_{i}, i=1,2,3$, are disjoint open sets and meas $\left(\Gamma_{1}\right)$ $>0$. The body is subjected to volume forces of density $f_{1}$, and prescribed zero displacements and tractions $f_{2}$ on $\Gamma_{1}$ and $\Gamma_{2}$, respectively. On $\Gamma_{3}$ the body is in unilateral contact with an obstacle following a version of nonlocal friction law where the coefficient of friction depends on the slip displacement.

Under these conditions, the classical formulation of the mechanical problem is the following.

Problem $P_{1}$. Find a displacement field $u: \Omega \rightarrow \mathbb{R}^{d}$ such that

$$
\operatorname{div} \sigma(u)=-f_{1} \quad \text { in } \Omega,
$$




$$
\begin{aligned}
& \sigma(u)=F \varepsilon(u) \quad \text { in } \Omega \text {, } \\
& u=0 \quad \text { on } \Gamma_{1} \text {, } \\
& \sigma \nu=f_{2} \quad \text { on } \Gamma_{2}, \\
& u_{\nu} \leq 0, \quad \sigma_{\nu}(u) \leq 0, \quad \sigma_{\nu}(u) u_{\nu}=0 \quad \text { on } \Gamma_{3}, \\
& \left|\sigma_{\tau}\right| \leq \mu\left(\left|u_{\tau}\right|\right)\left|R \sigma_{\nu}\right| \\
& \left.\begin{array}{l}
\left|\sigma_{\tau}\right|<\mu\left(\left|u_{\tau}\right|\right)\left|R \sigma_{\nu}\right| \Rightarrow u_{\tau}=0 \\
\left|\sigma_{\tau}\right|=\mu\left(\left|u_{\tau}\right|\right)\left|R \sigma_{\nu}\right| \Rightarrow \exists \lambda \geq 0: \sigma_{\tau}=-\lambda u_{\tau}
\end{array}\right\} \text { on } \Gamma_{3} .
\end{aligned}
$$

Here (2.1) represents the equilibrium equation where $\sigma=\sigma(u)$ denotes the stress tensor, while (2.2) is the elastic constitutive law in which $\varepsilon(u)$ is the small strain and $F$ is a given nonlinear function.

Equations (2.3) and (2.4) are the displacement and traction boundary conditions, respectively, in which $\nu$ denotes the unit outward normal vector on $\Gamma$ and $\sigma \nu$ represents the Cauchy stress vector.

Condition (2.5) is the classical Signorini contact condition without a gap and (2.6) represents a version of Coulomb's law of dry friction in which $\mu$ depends on the displacement $u_{\tau}$.

We note that in $(2.5), \sigma_{\nu}$ denotes the normal stress and $u_{\nu}$ is the normal displacement. When $u_{\nu}<0$, i.e. when there is separation between the body and the obstacle, condition (2.5) shows that $\sigma_{\nu}=0$; when $u_{\nu}=0$, i.e. when there is contact, then $\sigma_{\nu}<0$. In (2.6) the tangential shear cannot exceed the maximal frictional resistance $\mu\left(\left|u_{\tau}\right|\right)\left|R \sigma_{\nu}(u)\right|$.

Next, in the study of Problem $P_{1}$ we shall adopt the following notation and hypotheses:

We denote by $S_{d}$ the space of second order symmetric tensors on $\mathbb{R}^{d}$ $(d=2,3)$, while ' $'$ and $|\cdot|$ will represent the inner product and Euclidean norm on $S_{d}$ and $\mathbb{R}^{d}$, respectively, i.e.

$$
\begin{aligned}
& u . v=u_{i} v_{i}, \quad|v|=(v \cdot v)^{1 / 2} \quad \forall u, v \in \mathbb{R}^{d}, \\
& \sigma . \tau=\sigma_{i j} \tau_{i j}, \quad|\tau|=(\tau . \tau)^{1 / 2} \quad \forall \sigma, \tau \in S_{d} .
\end{aligned}
$$

Here and below the indices $i$ and $j$ run between 1 and $d$, and the summation convention over repeated indices is adopted.

To proceed with the variational formulation, we need some function spaces:

$$
\begin{aligned}
H & =\left(L^{2}(\Omega)\right)^{d}, \\
Q & =\left\{\tau=\left(\tau_{i j}\right): \tau_{i j}=\tau_{j i} \in L^{2}(\Omega)\right\}, \\
H_{1} & =\left(H^{1}(\Omega)\right)^{d} .
\end{aligned}
$$

$H, Q$ are Hilbert spaces equipped with the respective inner products:

$$
(u, v)_{H}=\int_{\Omega} u_{i} v_{i} d x, \quad\langle\sigma, \tau\rangle_{Q}=\int_{\Omega} \sigma_{i j} \tau_{i j} d x .
$$


The linearized strain tensor is defined as

$$
\varepsilon(v)=\left(\varepsilon_{i j}(v)\right)=\left(\frac{1}{2}\left(v_{i, j}+v_{j, i}\right)\right) \quad \forall v \in H_{1} .
$$

For every element $v \in H_{1}$, we also write $v$ for the trace of $v$ on $\Gamma$ and we denote by $v_{\nu}$ and $v_{\tau}$ the normal and the tangential components of $v$ on the boundary $\Gamma$ given by $v_{\nu}=v \cdot \nu, v_{\tau}=v-v_{\nu} \nu$. Similarly, $\sigma_{\nu}$ and $\sigma_{\tau}$ denote the normal and the tangential traces of a function

$$
\sigma \in Q_{1}=\{\tau \in Q: \operatorname{div} \tau \in H\} .
$$

When $\sigma$ is a regular function, we have

$$
\sigma_{\nu}=(\sigma \nu) . \nu, \quad \sigma_{\tau}=\sigma \nu-\sigma_{\nu} \nu,
$$

and the following Green's formula holds:

$$
\langle\sigma, \varepsilon(v)\rangle_{Q}+(\operatorname{div} \sigma, v)_{H}=\int_{\Gamma} \sigma \nu \cdot v d a \quad \forall v \in H_{1},
$$

where $d a$ is the measure surface element.

Next let $V$ be the closed subspace of $H_{1}$ defined by

$$
V=\left\{v \in H_{1}: v=0 \text { on } \Gamma_{1}\right\}
$$

and let the set of admissible displacements fields be given by

$$
K=\left\{v \in V: v_{\nu} \leq 0 \text { a.e. on } \Gamma_{3}\right\} .
$$

Since meas $\left(\Gamma_{1}\right)>0$, the following Korn's inequality holds [12]:

$$
\|\varepsilon(v)\|_{Q} \geq c_{\Omega}\|v\|_{H_{1}} \quad \forall v \in V
$$

where $c_{\Omega}>0$ is a constant which depends only on $\Omega$ and $\Gamma_{1}$. We equip $V$ with the inner product given by

$$
(u, v)_{V}=\langle\varepsilon(u), \varepsilon(v)\rangle_{Q}
$$

and let $\|\cdot\|_{V}$ be the associated norm. It follows from (2.7) that the norms $\|\cdot\|_{H_{1}}$ and $\|\cdot\|_{V}$ are equivalent and $\left(V,\|\cdot\|_{V}\right)$ is a real Hilbert space. Moreover, by Sobolev's trace theorem, there exists a constant $d_{\Omega}>0$ depending only on the domain $\Omega, \Gamma_{1}$ and $\Gamma_{3}$ such that

$$
\|v\|_{\left(L^{2}\left(\Gamma_{3}\right)\right)^{d}} \leq d_{\Omega}\|v\|_{V} \quad \forall v \in V .
$$

In the study of the mechanical problem $P_{1}$, we assume that the operator of elasticity $F$ satisfies 


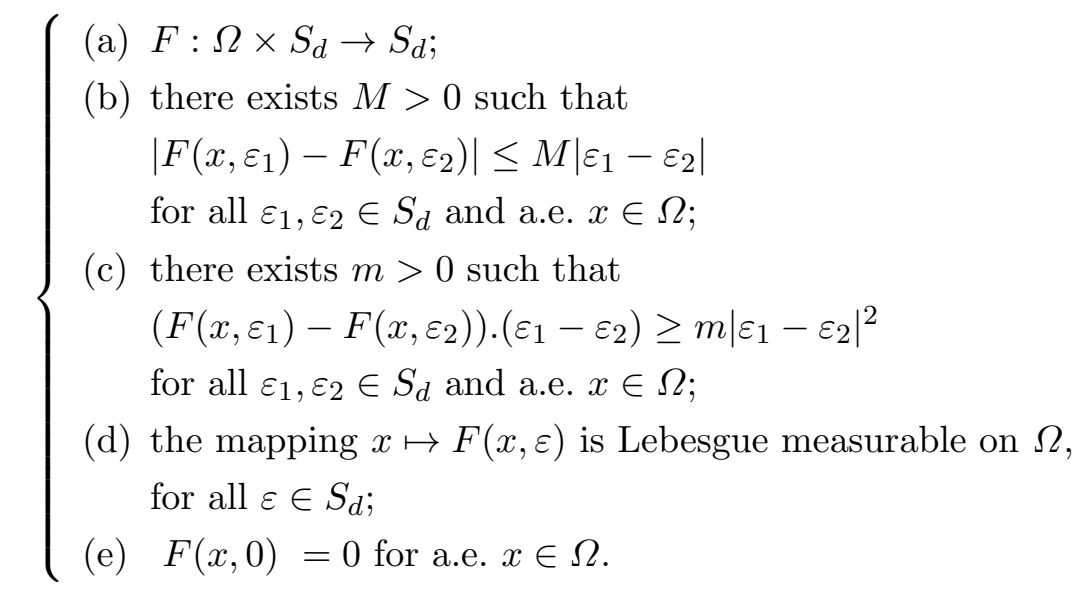

The forces and the tractions are assumed to satisfy

$$
f_{1} \in H, \quad f_{2} \in\left(L^{2}\left(\Gamma_{2}\right)\right)^{d},
$$

and we denote by $f$ the element of $V$ given by

$$
(f, v)_{V}=\int_{\Omega} f_{1} \cdot v d x+\int_{\Gamma_{2}} f_{2} \cdot v d a \quad \forall v \in V .
$$

We assume that the coefficient of friction $\mu$ satisfies

$$
\left\{\begin{aligned}
& \text { (a) } \mu: \Gamma_{3} \times \mathbb{R}_{+} \rightarrow \mathbb{R}_{+} ; \\
& \text {(b) there exists } L_{\mu}>0 \text { such that } \\
&|\mu(x, u)-\mu(x, v)| \leq L_{\mu}|u-v| \\
& \text { for all } u, v \in \mathbb{R}_{+} \text {and a.e. } x \in \Gamma_{3} ; \\
& \text { (c) } \text { there exists } \mu_{0}>0 \text { such that } \\
& \mu(x, u) \leq \mu_{0} \text { for all } u \in \mathbb{R}_{+} \text {and a.e. } x \in \Gamma_{3} ; \\
& \text { (d) } \text { the function } x \mapsto \mu(x, u) \text { is Lebesgue measurable on } \Gamma_{3} \\
& \\
& \quad \text { for all } u \in \mathbb{R}_{+} .
\end{aligned}\right.
$$

$R: H^{-1 / 2}(\Gamma) \rightarrow L^{2}\left(\Gamma_{3}\right)$ is a continuous linear operator (see [6]), that is, there exists a positive constant $c_{R}$ such that

$$
\|R \tau\|_{L^{2}\left(\Gamma_{3}\right)} \leq c_{R}\|\tau\|_{H^{-1 / 2}(\Gamma)} \quad \forall \tau \in H^{-1 / 2}(\Gamma) .
$$

Next, we define a subset $W$ of $H_{1}$ as

$$
W=\left\{v \in H_{1}: \operatorname{div} \sigma(v) \in H\right\}
$$

and let $j: W \times V \rightarrow \mathbb{R}$ be the functional defined by

$$
j(v, w)=\int_{\Gamma_{3}} \mu\left(\left|v_{\tau}\right|\right)\left|R \sigma_{\nu}(v)\right|\left|w_{\tau}\right| d a \quad \forall(v, w) \in W \times V .
$$


We note that if $v \in W$ then $\sigma(v)$ belongs to $Q_{1}$ and $\sigma_{\nu}(v)$ is an element of $H^{-1 / 2}(\Gamma)$. Thus, $j(v, \cdot)$ makes sense.

Now, using Green's formula, it is straightforward to see that if $u$ is a sufficiently regular function which satisfies (2.1)-(2.6), we obtain the following variational formulation of the mechanical problem $P_{1}$.

Problem $P_{2}$. Find a displacement field $u \in K \cap W$ such that

$$
\langle F \varepsilon(u), \varepsilon(v-u)\rangle_{Q}+j(u, v)-j(u, u) \geq(f, v-u)_{V} \quad \forall v \in K .
$$

3. Existence and uniqueness of solution. The main result of this section is the existence and uniqueness theorem for the weak formulation $P_{2}$ :

TheOREM 3.1. Let (2.9)-(2.12) hold. If

$$
\begin{aligned}
L_{\mu} c_{R} \frac{d_{\Omega}^{2}}{m}\left((M / m+1)\left\|f_{1}\right\|_{H} / c_{\Omega}+\left(d_{\Omega} M / m\right)\left\|f_{2}\right\|_{\left(L\left(\Gamma_{2}\right)\right)^{d}}\right) & \\
& +\mu_{0} c_{R} M \frac{d_{\Omega}}{m}<1,
\end{aligned}
$$

then Problem $P_{2}$ has a unique solution.

Proof. The proof will be carried out in several steps. It is based on fixed point arguments.

Let $g \in L_{+}^{2}\left(\Gamma_{3}\right)$ where $L_{+}^{2}\left(\Gamma_{3}\right)$ is the nonempty closed subset of $L^{2}\left(\Gamma_{3}\right)$ defined as

$$
L_{+}^{2}\left(\Gamma_{3}\right)=\left\{s \in L^{2}\left(\Gamma_{3}\right): s \geq 0 \text { a.e. on } \Gamma_{3}\right\}
$$

and let the functional $j_{g}: V \rightarrow \mathbb{R}$ be given by

$$
j_{g}(v)=\int_{\Gamma_{3}} g\left|v_{\tau}\right| d a \quad \forall v \in V .
$$

We now consider the following contact problem with given friction.

Problem $P_{g}$. Find $u_{g} \in K$ such that

$$
\left\langle F \varepsilon\left(u_{g}\right), \varepsilon\left(v-u_{g}\right)\right\rangle_{Q}+j_{g}(v)-j_{g}\left(u_{g}\right) \geq\left(f, v-u_{g}\right)_{V} \quad \forall v \in K .
$$

We prove the following lemma.

Lemma 3.2. For any $g \in L_{+}^{2}\left(\Gamma_{3}\right)$, Problem $P_{g}$ has a unique solution.

Proof. Let $A: V \rightarrow V$ be the operator given by $(A u, v)_{V}=\langle F \varepsilon(u), \varepsilon(v)\rangle_{Q}$ for $u, v \in V$. We use (2.8) and (2.9)(b) \& (c) to show that $A$ is strongly monotone and Lipschitz continuous. The functional $j_{g}: V \rightarrow \mathbb{R}$ is a continuous seminorm; since $K$ is a nonempty closed convex subset of $V$, it follows from the theory of elliptic variational inequalities (see [5]) that the inequality (3.2) has a unique solution.

We now consider the mapping $\Psi: L_{+}^{2}\left(\Gamma_{3}\right) \rightarrow L_{+}^{2}\left(\Gamma_{3}\right)$ defined as

$$
\Psi(g)=\mu\left(\left|u_{g \tau}\right|\right)\left|R \sigma_{\nu}\left(u_{g}\right)\right| \quad \forall g \in L_{+}^{2}\left(\Gamma_{3}\right) .
$$


We have the following result.

LEMma 3.3. If (3.1) holds, then the mapping $\Psi$ admits a unique fixed point $g^{*}$ and $u_{g^{*}}$ is a unique solution of Problem $P_{2}$.

Proof. Let $g_{1}, g_{2} \in L_{+}^{2}\left(\Gamma_{3}\right)$. Thus, by (2.12) and Green's formula,

$$
\left\|R\left(\sigma_{\nu}\left(u_{g_{1}}\right)\right)-R\left(\sigma_{\nu}\left(u_{g_{2}}\right)\right)\right\|_{L^{2}\left(\Gamma_{3}\right)} \leq c_{R} M\left\|u_{g_{1}}-u_{g_{2}}\right\|_{V} .
$$

We take $v=u_{g_{2}}$ in the inequality equivalent to (3.2) with $g=g_{1}, v=u_{g_{1}}$ in the inequality equivalent to (3.2) with $g=g_{2}$ and add the results to obtain

$$
\left\langle F \varepsilon\left(u_{g_{1}}\right)-F \varepsilon\left(u_{g_{2}}\right), \varepsilon\left(u_{g_{1}}\right)-\varepsilon\left(u_{g_{2}}\right)\right\rangle_{Q} \leq \int_{\Gamma_{3}}\left(g_{1}-g_{2}\right)\left(\left|u_{g_{2 \tau}}\right|-\left|u_{g_{1 \tau}}\right|\right) d a .
$$

Therefore using (2.8) we deduce from (3.5) that

$$
\left\|u_{g_{1}}-u_{g_{2}}\right\|_{V} \leq \frac{d_{\Omega}}{m}\left\|g_{1}-g_{2}\right\|_{L^{2}\left(\Gamma_{3}\right)} .
$$

Hence applying (3.4), we get

$$
\left.\| R\left(\sigma_{\nu}\left(u_{g_{1}}\right)\right)-R\left(\sigma_{\nu}\left(u_{g_{2}}\right)\right)\right)\left\|_{L^{2}\left(\Gamma_{3}\right)} \leq c_{R} M \frac{d_{\Omega}}{m}\right\| g_{1}-g_{2} \|_{L^{2}\left(\Gamma_{3}\right)}
$$

and furthermore using (2.8), (2.12) and (3.2), one obtains

$$
\begin{aligned}
& \left\|\Psi\left(g_{1}\right)-\Psi\left(g_{2}\right)\right\|_{L^{2}\left(\Gamma_{3}\right)}=\left\|\mu\left(\left|u_{g_{1} \tau}\right|\right)\left|R \sigma_{\nu}\left(u_{g_{1}}\right)\right|-\mu\left(\left|u_{g_{2} \tau}\right|\right)\left|R \sigma_{\nu}\left(u_{g_{2}}\right)\right|\right\|_{L^{2}\left(\Gamma_{3}\right)} \\
& =\|\left(\mu\left(\left|u_{g_{1} \tau}\right|\right)-\mu\left(\left|u_{g_{2} \tau}\right|\right)\right)\left|R \sigma_{\nu}\left(u_{g_{1}}\right)\right| \\
& +\mu\left(\left|u_{g_{2} \tau}\right|\right)\left(\left|R \sigma_{\nu}\left(u_{g_{1}}\right)\right|-\left|R \sigma_{\nu}\left(u_{g_{2}}\right)\right|\right) \|_{L^{2}\left(\Gamma_{3}\right)} \\
& \leq\left[L_{\mu} c_{R} \frac{d_{\Omega}^{2}}{m}\left((M / m+1)\left\|f_{1}\right\|_{H} / c_{\Omega}+\left(d_{\Omega} M / m\right)\left\|f_{2}\right\|_{\left(L\left(\Gamma_{2}\right)\right)^{d}}\right)+\mu_{0} c_{R} M \frac{d_{\Omega}}{m}\right] \\
& \times\left\|g_{1}-g_{2}\right\|_{L^{2}\left(\Gamma_{3}\right)} .
\end{aligned}
$$

Thus if (3.1) holds, the mapping $\Psi$ is a contraction. It admits a unique fixed point $g_{*}$ and $u_{g^{*}}$ is a unique solution to inequality (2.13).

Next denote $u_{g^{*}}=u$.

Remark 3.4. As $u_{g} \in W$ for all $g \in L_{+}^{2}\left(\Gamma_{3}\right)$, it follows that $u \in W$.

4. Finite element approximation. In this section we study the finite element approximation of the variational problem $P_{1}$. Suppose $\Omega$ is a polygonal domain in $\mathbb{R}^{d}$ and let $V_{h} \subset V$ be a finite element subspace where $h \rightarrow 0_{+}$. We define a nonempty closed convex set $K_{h}$ by

$$
K_{h}=\left\{v_{h} \in V_{h}: v_{h \nu} \leq 0 \text { a.e. on } \Gamma_{3}\right\} .
$$

We note that $K_{h} \subset K$ and formulate a discrete problem: 
Problem $P_{h}$. Find $u_{h} \in K_{h} \cap W$ such that

$$
\begin{aligned}
\left\langle F \varepsilon\left(u_{h}\right), \varepsilon\left(v_{h}-u_{h}\right)\right\rangle_{Q}+j\left(u_{h}, v_{h}\right)- & j\left(u_{h}, u_{h}\right) \\
& \geq\left(f, v_{h}-u_{h}\right)_{V} \quad \forall v_{h} \in K_{h} .
\end{aligned}
$$

It is clear that under the assumptions of Theorem 3.1 and condition (3.1), Problem $P_{h}$ has a unique solution $u_{h} \in K_{h}$.

Now we shall establish the following Cea-type inequality.

Theorem 4.1. Assume (2.9)-(2.12) and (3.1) hold. Then there exists a constant $c>0$ independent of $h$ such that

$$
\left\|u-u_{h}\right\|_{V} \leq c \inf _{v_{v} \in V_{h}}\left(\left\|u-v_{h}\right\|_{V}+\left\|u-v_{h}\right\|_{V}^{1 / 2}+\left\|u-v_{h}\right\|_{\left(L^{2}\left(\Gamma_{3}\right)\right)^{d}}^{1 / 2}\right) .
$$

Proof. Using (2.9)(c) we have

$$
m\left\|u-u_{h}\right\|_{V}^{2} \leq\left(A u-A u_{h}, u-u_{h}\right)_{V} .
$$

On the other hand, for any $v_{h} \in K_{h}$ we have $\left(A u-A u_{h}, u-u_{h}\right)_{V} \leq\left(A u-A u_{h}, u-v_{h}\right)_{V}+\left(A u, v_{h}-u_{h}\right)_{V}-\left(A u_{h}, v_{h}-u_{h}\right)_{V}$, hence

$$
\begin{aligned}
& m\left\|u-u_{h}\right\|_{V}^{2} \\
& \quad \leq\left(A u-A u_{h}, u-v_{h}\right)_{V}+\left(A u, v_{h}-u_{h}\right)_{V}-\left(A u_{h}, v_{h}-u_{h}\right)_{V} .
\end{aligned}
$$

We now use (2.13) with $v=u_{h}$ and (4.1) to find that

$$
\begin{aligned}
\left(A u, v_{h}-u_{h}\right)_{V}-\left(A u_{h}, v_{h}-u_{h}\right)_{V} \leq\left(A u, v_{h}-u\right)_{V} \\
+j\left(u_{h}, v_{h}\right)-j\left(u_{h}, u_{h}\right)+j\left(u, u_{h}\right)-j(u, u)+\left(f, u-v_{h}\right)_{V} .
\end{aligned}
$$

From (4.3) and (4.4) we obtain

$$
m\left\|u-u_{h}\right\|_{V}^{2} \leq A_{1}+A_{2}+A_{3}+A_{4},
$$

where

$$
\begin{aligned}
& A_{1}=\left(A u-A u_{h}, u-v_{h}\right)_{V} \\
& A_{2}=\left(A u, v_{h}-u\right)_{V}+j\left(u, v_{h}\right)-j(u, u)-\left(f, v_{h}-u\right)_{V}, \\
& A_{3}=j\left(u, u_{h}\right)-j\left(u_{h}, u_{h}\right)+j\left(u_{h}, u\right)-j(u, u), \\
& A_{4}=j\left(u_{h}, v_{h}\right)-j\left(u, v_{h}\right)+j(u, u)-j\left(u_{h}, u\right) .
\end{aligned}
$$

Now we estimate each term $A_{i}, i=1, \ldots, 4$. First,

$$
\left|A_{1}\right| \leq M\left\|u-u_{h}\right\|_{V}\left\|u-v_{h}\right\|_{V} \text {. }
$$

The second term $A_{2}$ can be estimated as follows. We have

$$
\begin{aligned}
A_{2}= & \int_{\Omega} F \varepsilon(u) \cdot \varepsilon\left(v_{h}-u\right) d x+\int_{\Gamma_{3}} \mu\left(\left|u_{\tau}\right|\right)\left|R \sigma_{\nu}(u)\right|\left(\left|v_{h \tau}\right|-\left|u_{\tau}\right|\right) d a \\
& -\left(f, v_{h}-u\right)_{V} .
\end{aligned}
$$


Using the properties of $R$ and $\mu$, we get

$$
\begin{aligned}
\left|A_{2}\right| \leq & \left(|F \varepsilon(u)|_{Q}+\|f\|_{V}\right)\left\|u-v_{h}\right\|_{V} \\
& +\mu_{0}\left\|R \sigma_{\nu}(u)\right\|_{L^{2}\left(\Gamma_{3}\right)}\left\|u-v_{h}\right\|_{\left(L^{2}\left(\Gamma_{3}\right)\right)^{d}} .
\end{aligned}
$$

In the same way we estimate the term $A_{3}$ as

$$
\begin{aligned}
& \quad\left|A_{3}\right| \leq \\
& \left.\left[L_{\mu} c_{R} d_{\Omega}^{2}\left((M / m+1)\left\|f_{1}\right\|_{H} / c_{\Omega}+\left(d_{\Omega} M / m\right)\left\|f_{2}\right\|_{\left(L\left(\Gamma_{2}\right)\right)^{d}}\right)+\mu_{0} c_{R} M d_{\Omega}\right)\right] \\
& \times\left\|u-u_{h}\right\|_{V}^{2} .
\end{aligned}
$$

Finally, to estimate the last term $A_{4}$, we use again the properties of $R$ and $\mu$ and to obtain

$$
\begin{aligned}
\left|A_{4}\right|= & \left|j\left(u_{h}, v_{h}\right)-j\left(u_{h}, u\right)+j(u, u)-j\left(u, v_{h}\right)\right| \\
= & \left|\int_{\Gamma_{3}}\left(\mu\left(\left|u_{\tau}\right|\right)\left|R \sigma_{\nu}(u)\right|-\mu\left(\mid u_{h \tau}\right)\left|R \sigma_{\nu}\left(u_{h}\right)\right|\right)\left(\left|u_{\tau}\right|-\left|v_{h \tau}\right|\right) d a\right| \\
\leq & \int_{\Gamma_{3}}\left|\left(\mu\left(\left|u_{\tau}\right|\right)\left|R \sigma_{\nu}(u)\right|-\mu\left(\left|u_{h \tau}\right|\right)\left|R \sigma_{\nu}\left(u_{h}\right)\right|\right)\left(\left|u_{\tau}\right|-\left|v_{h \tau}\right|\right)\right| d a \\
\leq & \int_{\Gamma_{3}} \mu\left(\left|u_{h \tau}\right|\right)|| R \sigma_{\nu}(u)|-| R \sigma_{\nu}\left(u_{h}\right)|| \cdot\left|u-v_{h}\right| d a \\
& \quad+\int_{\Gamma_{3}}\left(\mu\left(\left|u_{\tau}\right|\right)-\mu\left(\left|u_{h \tau}\right|\right)\right)\left|R \sigma_{\nu}(u)\right| \cdot\left|u-v_{h}\right| d a \\
\leq & \mu_{0} c_{R} d_{\Omega}\left(M+\|f\|_{V}\right)\left\|u-u_{h}\right\|_{V}\left\|u-v_{h}\right\|_{V} \\
& \quad+2 \mu_{0}\left\|R \sigma_{\nu}(u)\right\|_{L^{2}\left(\Gamma_{3}\right)}\left\|u-v_{h}\right\|_{\left(L^{2}\left(\Gamma_{3}\right)\right)^{d}},
\end{aligned}
$$

and so

$$
\begin{aligned}
\left|A_{4}\right| \leq & \mu_{0} c_{R} d_{\Omega} M\left\|u-u_{h}\right\|_{V}\left\|u-v_{h}\right\|_{V} \\
& +2 \mu_{0}\left\|R \sigma_{\nu}(u)\right\|_{L^{2}\left(\Gamma_{3}\right)}\left\|u-v_{h}\right\|_{\left(L^{2}\left(\Gamma_{3}\right)\right)^{d}} .
\end{aligned}
$$

Now to end the proof we use (3.1), (4.5)-(4.8) and the elementary inequality

$$
a b \leq \delta a^{2}+\frac{1}{4 \delta} b^{2} \quad \forall \delta>0 .
$$

Hence we obtain an inequality of the form (4.2).

Now to derive an optimal error estimate, we assume in addition that

$$
u \in\left(H^{2}(\Omega)\right)^{d},
$$

and use linear elements for the finite element space $V_{h}$. We need the follow- 
ing standard interpolation error estimates (see [7]):

$$
\begin{aligned}
& \left\|u-\pi_{h} u\right\|_{V} \leq c h\|u\|_{\left(H^{2}(\Omega)\right)^{d}}, \\
& \left\|u-\pi_{h} u\right\|_{\left(L^{2}\left(\Gamma_{3}\right)\right)^{d}} \leq c h^{3 / 2}\|u\|_{\left(H^{2}(\Omega)\right)^{d}},
\end{aligned}
$$

where $\pi_{h} u$ denotes the $V_{h}$-interpolant of the function $u$. Therefore, using the regularity (4.9), we deduce from (4.2) the error estimate

$$
\left\|u-u_{h}\right\|_{V} \leq c h^{1 / 2} \text {. }
$$

However, following [6] we remark that this type of error estimate is not optimal. In order to obtain an optimal error estimate we assume an additional regularity for the solution.

TheOREM 4.2. Let the hypotheses of Theorem 4.1 hold and, in addition, suppose that $\sigma \nu \in\left(L^{2}\left(\Gamma_{3}\right)\right)^{d}$. Then there exists a constant $c>0$ independent of $h$ such that

$$
\left\|u-u_{h}\right\|_{V} \leq c \inf _{v_{h} \in K_{h}}\left(\left\|u-v_{h}\right\|_{V}+\left\|u-v_{h}\right\|_{\left(L^{2}\left(\Gamma_{3}\right)\right)^{d}}^{1 / 2}\right)
$$

where $c$ is a positive constant independent of $h$.

Proof. We see that it suffices to provide an estimate of $A_{2}$. Indeed, using Green's formula we have

$$
\begin{aligned}
\left|A_{2}\right| \leq & \left|\int_{\Gamma_{3}} \sigma_{\nu}\left(v_{h \nu}-u_{\nu}\right) d a+\int_{\Gamma_{3}} \sigma_{\tau} \cdot\left(v_{h \tau}-u_{\tau}\right) d a\right| \\
& +\left|\int_{\Gamma_{3}} \mu\left(\left|u_{\tau}\right|\right)\right| R \sigma_{\nu}(u)\left|\left(\left|v_{h \tau}\right|-\left|u_{\tau}\right|\right) d a\right| \\
\leq & \left(\left\|\sigma_{\nu}\right\|_{L^{2}\left(\Gamma_{3}\right)}+\left\|\sigma_{\tau}\right\|_{\left(L^{2}\left(\Gamma_{3}\right)\right)^{d}}+\mu_{0}\left\|R \sigma_{\nu}(u)\right\|_{L^{2}\left(\Gamma_{3}\right)}\right)\left\|u-v_{h}\right\|_{\left(L^{2}\left(\Gamma_{3}\right)\right)^{d}} .
\end{aligned}
$$

Hence if we take $c \geq\left\|\sigma_{\nu}\right\|_{L^{2}\left(\Gamma_{3}\right)}+\left\|\sigma_{\tau}\right\|_{\left(L^{2}\left(\Gamma_{3}\right)\right)^{d}}+\mu_{0}\left\|R \sigma_{\nu}(u)\right\|_{L^{2}\left(\Gamma_{3}\right)}$ we conclude the proof.

Now to derive an optimal error estimate, we assume in addition that

$$
u \in\left(H^{2}(\Omega)\right)^{d},\left.\quad u\right|_{\Gamma_{3}} \in\left(H^{2}\left(\Gamma_{3}\right)\right)^{d} .
$$

We again need the following standard interpolation error estimates

$$
\begin{aligned}
& \left\|u-\pi_{h} u\right\|_{V} \leq c_{1} h\|u\|_{\left(H^{2}(\Omega)\right)^{d}}, \\
& \left\|u-\pi_{h} u\right\|_{\left(L^{2}\left(\Gamma_{3}\right)\right)^{d}} \leq c_{1} h^{2}\|u\|_{\left(H^{2}\left(\Gamma_{3}\right)\right)^{d}} .
\end{aligned}
$$

Therefore, using the regularity (4.11), we deduce from (4.10) the optimal error estimate

$$
\left\|u-u_{h}\right\|_{V} \leq c h .
$$

Next the finite element system (4.1) can be approximated by a fixedpoint iterative method. Indeed, by choosing the initial guess $u_{h}^{0} \in K_{h} \cap W$ 
we define recursively a sequence $\left(u_{h}^{n}\right) \subset K_{h}$ by

$$
\begin{aligned}
\left\langle F \varepsilon\left(u_{h}^{n+1}\right), \varepsilon\left(v_{h}-u_{h}^{n+1}\right)\right\rangle_{Q}+j\left(u_{h}^{n}, v_{h}\right)-j\left(u_{h}^{n}, u_{h}^{n+1}\right) & \\
& \geq\left(f, v_{h}-u_{h}^{n+1}\right)_{V} \quad \forall v_{h} \in K_{h} .
\end{aligned}
$$

We have the following theorem.

TheOREM 4.3. Suppose the assumptions of Theorem 3.1 hold. Then the iterative method (4.11) converges:

$$
\left\|u_{h}^{n}-u_{h}\right\|_{V} \rightarrow 0 \quad \text { as } n \rightarrow \infty .
$$

Moreover, there exists a constant $k \in] 0,1[$ such that

$$
\left\|u_{h}^{n}-u_{h}\right\|_{V} \leq c k^{n} .
$$

Proof. We take $v_{h}=u_{h}^{n+1}$ in (3.1), then take $v_{h}=u_{h}$ in (4.11), after adding the resulting inequalities we obtain

$$
\begin{aligned}
\left\langle F \varepsilon\left(u_{h}\right)-\right. & \left.F \varepsilon\left(u_{h}^{n+1}\right), \varepsilon\left(u_{h}-u_{h}^{n+1}\right)\right\rangle_{Q} \\
& \leq j\left(u_{h}, u_{h}^{n+1}\right)-j\left(u_{h}, u_{h}\right)+j\left(u_{h}^{n}, u_{h}\right)-j\left(u_{h}^{n}, u_{h}^{n+1}\right) .
\end{aligned}
$$

Now by the same arguments used in the proof of Theorem 4.1 we find from (4.14) that

$$
\begin{aligned}
\leq & {\left[L_{\mu} c_{R} \frac{d_{\Omega}^{2}}{m}\left((M / m+1)\left\|f_{1}\right\|_{H} / c_{\Omega}+\left(d_{\Omega} M / m\right)\left\|f_{2}\right\|_{\left(L\left(\Gamma_{2}\right)\right)^{d}}\right)+\mu_{0} c_{R} M \frac{d_{\Omega}}{m}\right] } \\
& \times\left\|u_{h}^{n}-u_{h}\right\|_{V} .
\end{aligned}
$$

Then if we let $k$ denote the quantity in the square brackets, we immediately conclude that (4.15) implies (4.13) and then (4.12).

\section{References}

[1] M. Barboteu, X. Cheng, and M. Sofonea, Analysis of a contact problem with unilateral constraint and slip-dependent friction, Math. Mech. Solids (2014) (online).

[2] Z. Belhachmi and F. Ben Belgacem, Quadratic finite element approximation of the Signorini problem, Math. Comp. 72 (2003), 83-104.

[3] F. Ben Belgacem, Numerical simulation of some variational inequalities arisen from unilateral contact problems by the finite element mehods, SIAM J. Numer. Anal. 37 (2000), 1198-1216.

[4] F. Ben Belgacem and Y. Renard, Hybrid finite element methods for the Signorini problem, Math. Comp. 72 (2003), 1117-1145.

[5] H. Brézis, Equations et inéquations non linéaires dans les espaces vectoriels en dualité, Ann. Inst. Fourier (Grenoble) 18 (1968), no. 1, 115-175.

[6] A. Capatina-Radoslovescu and M. Cocu, Internal approximation of quasi-variational inequalities, Numer. Math. 59 (1991), 385-398. 
[7] P. G. Ciarlet, The Finite Element Methods for Elliptic Problems, North-Holland, 1978.

[8] C. Ciulcu, D. Motreanu and M. Sofonea, Analysis of an elastic contact problem with slip dependent coefficient of friction, Math. Inequal. Appl. 4 (2001), 465-479.

[9] M. Cocu, E. Pratt and M. Raous, Formulation and approximation of quasistatic frictional contact, Int. J. Engrg. Sci. 34 (1996), 783-798.

[10] C. Corneschi, T.-V. Horau-Mantel and M. Sofonea, A quasistatic contact problem with slip dependent coefficient of friction for elastic materials, J. Appl. Anal. 8 (2002), 63-82.

[11] G. Duvaut, Équilibre d'un solide élastique avec contact unilatéral et frottement de Coulomb, C. R. Acad. Sci. Paris 290 (1980), 263-265.

[12] G. Duvaut et J.-L. Lions, Les inéquations en mécanique et en physique, Dunod, Paris, 1972.

[13] C. Eck, J. Jarušek and M. Krbec, Unilateral Contact Problems. Variational Methods and Existence Theorems, Pure Appl. Math. 270, Chapman \& Hall/CRC, Boca Raton, FL, 2005.

[14] G. Glowinski, J.-L. Lions, et R. Tremolières, Analyse numérique des inéquations variationnelles, Tomes 1, 2, Dunod, Paris, 1976.

[15] N. Kikuchi and T. J. Oden, Contact Problems in Elasticity: A Study of Variational Inequalities and Finite Element Methods, SIAM, Philadelphia, 1988.

[16] J. T. Oden and E. Pires, Contact problems in elastostatics with non-local friction laws, TICOM Report 81-82, Univ. of Texas at Austin, 1981.

[17] A. Signorini, Sopra alcune questioni di elastostatica, Atti della Società Italiana per il Progresso delle Scienze 21 (1933), 143-148.

[18] M. Sofonea, W. Han and M. Shillor, Analysis and Approximation of Contact Problems with Adhesion or Damage, Pure Appl. Math. 276, Chapman \& Hall/CRC, Boca Raton, FL, 2006.

[19] A. Touzaline, A quasistatic unilateral contact problem with slip dependent coefficient of friction for nonlinear elastic materials, Electron. J. Differential Equations 2006, art. $144,14 \mathrm{pp}$.

Arezki Touzaline

Faculté de Mathématiques, USTHB

Laboratoire de Systèmes Dynamiques

BP 32 El Alia

Bab-Ezzouar, 16111, Algeria

E-mail: ttouzaline@yahoo.fr 